\title{
Biomechanical comparison of different graft positions for single-bundle anterior cruciate ligament reconstruction
}

\author{
Yuki Kato • Akira Maeyama · Pisit Lertwanich · Joon Ho Wang • \\ Sheila J. M. Ingham · Scott Kramer · Cesar Q. A. Martins • \\ Patrick Smolinski $\cdot$ Freddie H. Fu
}

Received: 29 May 2011/Accepted: 28 February 2012/Published online: 15 March 2012

(C) The Author(s) 2012. This article is published with open access at Springerlink.com

\begin{abstract}
Purpose Recent reports have highlighted the importance of an anatomic tunnel placement for anterior cruciate ligament (ACL) reconstruction. The purpose of this study was to compare the effect of different tunnel positions for single-bundle ACL reconstruction on knee biomechanics.

Methods Sixteen fresh-frozen cadaver knees were used. In one group ( $n=8)$, the following techniques were used for knee surgery: (1) anteromedial (AM) bundle reconstruction (AM-AM), (2) posterolateral (PL) bundle reconstruction (PL-PL) and (3) conventional vertical single-bundle reconstruction (PL-high AM). In the other group $(n=8)$, anatomic mid-position single-bundle reconstruction (MIDMID) was performed. A robotic/universal force-moment sensor system was used to test the knees. An anterior load of $89 \mathrm{~N}$ was applied for anterior tibial translation (ATT) at $0^{\circ}$, $15^{\circ}, 30^{\circ}$ and $60^{\circ}$ of knee flexion. Subsequently, a combined rotatory load $(5 \mathrm{Nm}$ internal rotation and $7 \mathrm{Nm}$ valgus moment) was applied at $0^{\circ}, 15^{\circ}, 30^{\circ}$ and $45^{\circ}$ of knee flexion. The ATT and in situ forces during the application of the external loads were measured.
\end{abstract}

Y. Kato · A. Maeyama - P. Lertwanich - J. H. Wang ·

S. J. M. Ingham - C. Q. A. Martins · P. Smolinski ·

F. H. Fu $(\bowtie)$

Department of Orthopaedic Surgery, University of Pittsburgh,

3471 Fifth Avenue, 1010 Kaufmann Building,

Pittsburgh, PA 15213, USA

e-mail: ffu@upmc.edu

Y. Kato

Department of Orthopaedic Surgery, Nihon University,

Tokyo, Japan

S. Kramer · P. Smolinski · F. H. Fu

Department of Mechanical Engineering,

University of Pittsburgh, Pittsburgh, PA, USA
Results Compared with the intact ACL, all reconstructed knees had a higher ATT under anterior load at all flexion angles and a lower in situ force during the anterior load at $60^{\circ}$ of knee flexion. In the case of combined rotatory loading, the highest ATT was achieved with PL-high AM; the in situ force was most closely restored with MIDMID, and the in situ force was the highest AM-AM at each knee flexion angle.

Conclusion Among the techniques, AM-AM afforded the highest in situ force and the least ATT.

Keywords Anterior cruciate ligament - Single-bundle . In situ force $\cdot$ Anterior tibial translation $\cdot$ Anatomic

\section{Introduction}

Anterior cruciate ligament (ACL) reconstruction is one of the most common orthopaedic procedures performed in the United States, with approximately 105,000 surgeries performed per year [23]. The trans-tibial approach has been described as the recommended method for femoral tunnel placement [31]; however, this procedure poses the risk of a high/vertical placement of the femoral tunnel in the intercondylar notch and a discrepancy between the tunnel position and the point of ACL insertion [2]. The trans-tibial technique for single-bundle ACL reconstruction achieves good-to-excellent results in only $60 \%$ of patients [6], and 20-30\% of athletes do not regain their previous level of performance [28]. To enhance its success rate, the conventional ACL reconstruction method needs to be further improved.

While double-bundle ACL reconstruction is gaining greater use, single-bundle reconstruction is still the most frequently performed method and is also useful when 
double-bundle reconstruction cannot be performed such as in cases with open growth plates, severe arthritic changes, multiple ligament injuries, a narrow notch, severe lateral femoral condyle bone bruising or tear of only one bundle [24]. The tibial ACL insertion site is a broad oval area, approximately $11 \mathrm{~mm}$ diameter in the coronal plane and $17 \mathrm{~mm}$ in the sagittal plane [1, 14]. Because of the fanning of the ligament, the tibial ACL insertion site is larger than the midsubstance and femoral attachment of the ligament [15]. With a single-bundle ACL reconstruction using a hamstrings graft, it is difficult to cover a large part of the ACL footprint with a round graft. Given this, little is known about the optimal tunnel position for anatomic single-bundle ACL reconstruction, although recent clinical studies recommend that the single-bundle graft be placed in the mid-bundle position of the ACL footprint [3].

To improve the single-bundle ACL reconstruction, while taking anatomic graft placement into account, the best tunnel positions must be evaluated. The purpose of this study was to compare the effect of different tunnel positions for single-bundle ACL reconstruction on knee biomechanics. It is hypothesized that the anatomic tunnel position is superior to the non-anatomic tunnel position and that the mid-bundle position is the most well balanced of the anatomic positions.

\section{Materials and methods}

Sample selection and grouping

Sixteen fresh-frozen cadaveric knees were used in the study. Each specimen was screened by a CT scan and a manual examination, and the existence of an intact ACL was confirmed by arthroscopy. Knees were excluded if evidence of any of the following was present: ligament injuries, previous knee surgery, osteoarthritis and bony abnormalities. All specimens were stored at $-20{ }^{\circ} \mathrm{C}$ and thawed at room temperature $24 \mathrm{~h}$ before the test. All soft tissues until a distance of approximately $10 \mathrm{~cm}$ away from both sides of the joint sides were removed. The exposed femur and tibia were secured in an epoxy compound (Bondo, Atlanta, Georgia, USA) for mounting in custommade aluminium clamps. The femoral side was rigidly fixed to a base, while the tibial side was mounted to the end-effector of the robot through a universal force-moment sensor (UFS), as shown in Fig. 1. The specimens were kept moist throughout the testing.

Four ACL reconstruction techniques were evaluated: (1) anatomic mid-bundle single-bundle reconstruction (MID-MID; $n=8$ ), with the graft being placed from the midpoint between the tibial AM and PL footprints to the midpoint between the femoral AM and PL footprints;

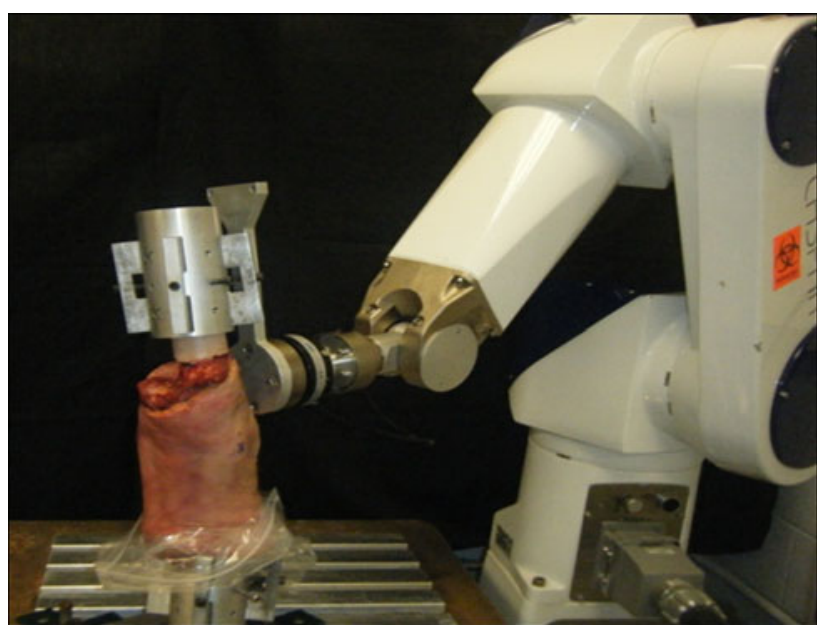

Fig. 1 The robotic testing system. The tibia and femur were secured in aluminium cylinders using epoxy compound and placed in the testing system. The tibial cylinder was connected to the universal force-moment sensor

(2) anatomic AM bundle single-bundle reconstruction (AM-AM; $n=8$ ), with the graft being placed from the tibial AM footprint to the femoral AM footprint; (3) anatomic PL bundle single-bundle reconstruction (PL-PL; $n=8$ ), with the graft being placed from the tibial PL footprint to the femoral PL footprint; and (4) conventional single-bundle reconstruction (PL-high AM; $n=8$ ), with the graft being placed from the tibial PL footprint to the femoral high AM position in the notch. Of the sixteen knees used, eight were used for MID-MID reconstructions, and the other eight knees were used for the remaining reconstructions (AM-AM, PL-PL and PL-high $\mathrm{AM}$ ) with the three reconstructions being done in each knee (Fig. 2). There was no significant difference in the age or sex between the MID-MID reconstructed knee group and the multiple-reconstructed knee group (AMAM, PL-PL and PL-high AM). The MID-MID reconstruction was performed in one group because it was difficult to place femoral tunnels at all positions (MID, PL, AM high AM) simultaneously; in particular, MID femoral tunnel creation between AM and PL femoral tunnels posed a risk of tunnel overlap.

\section{Interventions}

A 3-portal technique was used with anterolateral, anteromedial and accessory medial portals with a $30^{\circ}$ scope [13]. The two functional ACL bundles (AM and PL bundles) were differentiated by their tension patterns during knee motion. The ACL was removed with an electrothermal arthroscopy system (Vulcan, Smith and Nephew, Endoscopy, Andover, MA). After identifying the ACL foot print and removing the ACL, ACL reconstruction was performed. A guide wire (2.4 mm diameter) was inserted into 

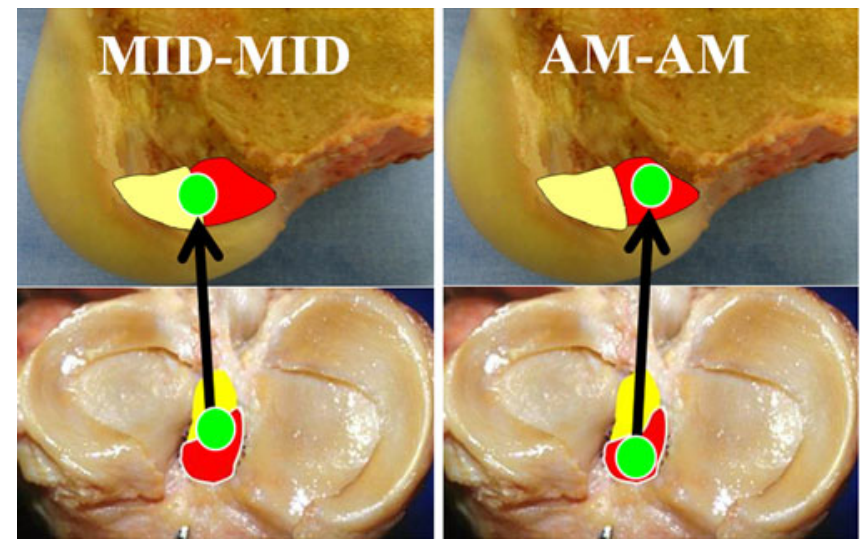

Fig. 2 Four different tunnel positions for single-bundle anterior cruciate ligament reconstructions. Three anatomic anterior cruciate ligament reconstructions (MID-MID, AM-AM and PL-PL) and one

the centre of each tibial ACL bundle footprint (AM and PL) from the anteromedial aspect of the tibia using a tibial drill guide system (Smith and Nephew Endoscopy, Andover, MA). The wire was then over drilled with a cannulated reamer (6 mm diameter). In 8 knees, three femoral tunnels (PL, AM and high AM) were created using a trans-portal technique [13]. A guide pin was inserted into the centre of the AM footprint, PL footprint and high AM position, which was located at the 11 or 1 o'clock position of the superoanterior portion of the ACL femoral footprint on the lateral wall of the intercondylar notch (Fig. 3a). The pin was then over drilled to the anterolateral femoral cortex by using a cannulated reamer ( $6 \mathrm{~mm}$ diameter). For the MIDMID reconstruction, the tibial and femoral tunnels were drilled between the centres of the AM and PL footprints of the tibia and femur in eight knees. Although one of the principles of an anatomic reconstruction is individualized surgery [28], $6 \mathrm{~mm}$ diameter bone tunnels were made for every reconstruction in this study to avoid AM and PL tunnel overlap (Fig. 3b). The knees were checked for the occurrence of tunnel overlap by CT scans after each test.

Previously harvested semitendinosus and gracilis tendons from human cadaver knees were used as grafts and were trimmed to a diameter of $6 \mathrm{~mm}$. A No.5 braised polyester suture was whip stitched with a tapered needle into the free ends of the folded grafts. The graft was passed through the extra-cortical button loop (EndoButton, Smith and Nephew, Endoscopy, Andover, MA) to make it double stranded. At graft fixation, an initial tension of $60 \mathrm{~N}$ load was applied (ligament tension meter; Meira Corp, Nagoya, Aichi, Japan) [9] at $0^{\circ}$ of knee flexion for the PL-PL reconstruction and at $30^{\circ}$ of knee flexion for the other reconstructions (MID-MID, AM-AM and PLhigh AM) $[11,16,35]$. Staples were used to fix the graft on the tibia.
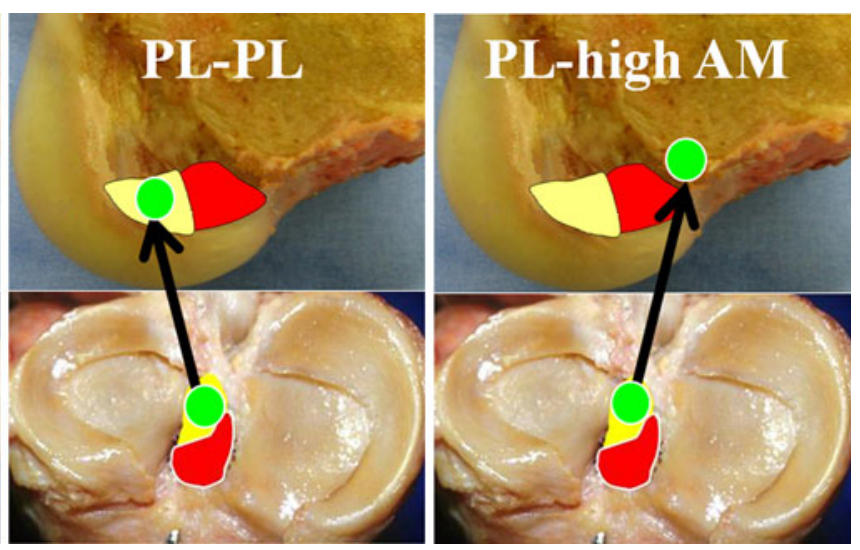

non-anatomic anterior cruciate ligament reconstruction (PL-high AM) were compared

\section{Outcome measurements}

The robotic/UFS testing system was used to determine knee joint kinematics and in situ forces of the ligament and the reconstruction graft $[27,32,33,34]$. The robotic manipulator (CASPAR, OrthoMaquet, Rastatt, Germany) is a six-joint, serial articulation device that allows 6-degree-of-freedom motion of the knee with repeatability of $0.02 \mathrm{~mm}$ at each joint according to the manufacturer. The UFS (model 4015; JR3 Inc, Woodland, California, USA) is capable of measuring 3 orthogonal forces and moments with repeatability of $0.2 \mathrm{~N}$ and $0.1 \mathrm{Nm}$, respectively, according to the manufacturer. A custom MATLAB program with a multitask operating system (Math Works Inc., Natick, Massachusetts, USA) was utilized to control the testing system, to monitor knee kinematics and calculate the in situ forces of the ACL and the reconstructed grafts. During the experiment, this testing system was operated in both the force- and displacement-control modes.

The specimen was initially mounted to the testing system at full extension of the knee (measured with a goniometer). The path of passive flexion-extension of the intact knee was determined with the robotic/UFS testing system by moving the tibia from full extension to $90^{\circ}$ of flexion by $0.5^{\circ}$ increments. At each incremental flexion of knee flexion, the forces and moments generated by the specimen in the remaining 5-degree-of-freedom were minimized by the iterative algorithm of the robot control software. The positions at full extension and $15^{\circ}, 30^{\circ}$ and $60^{\circ}$ of flexion were used as the starting positions for applying external tibial loads throughout the test. The following external loading conditions were applied to the tibia: (1) $89 \mathrm{~N}$ of anterior tibial load (simulated KT 1000 test) [36] at full extension and $15^{\circ}, 30^{\circ}$ and $60^{\circ}$ of flexion and (2) a combined $7 \mathrm{Nm}$ valgus torque and $5 \mathrm{Nm}$ internal tibial rotation 
Fig. 3 a Three femoral tunnels (PL, AM and high AM) and two tibial tunnels (PL and AM) were created in a multiplereconstructed knee (arthroscopic view and 3D CT scan reconstruction). b For the MID-MID reconstruction, the tibial and femoral tunnels were drilled between the centre of the $\mathrm{AM}$ and PL footprints of the tibia and femur (arthroscopic view and 3D CT scan reconstruction)

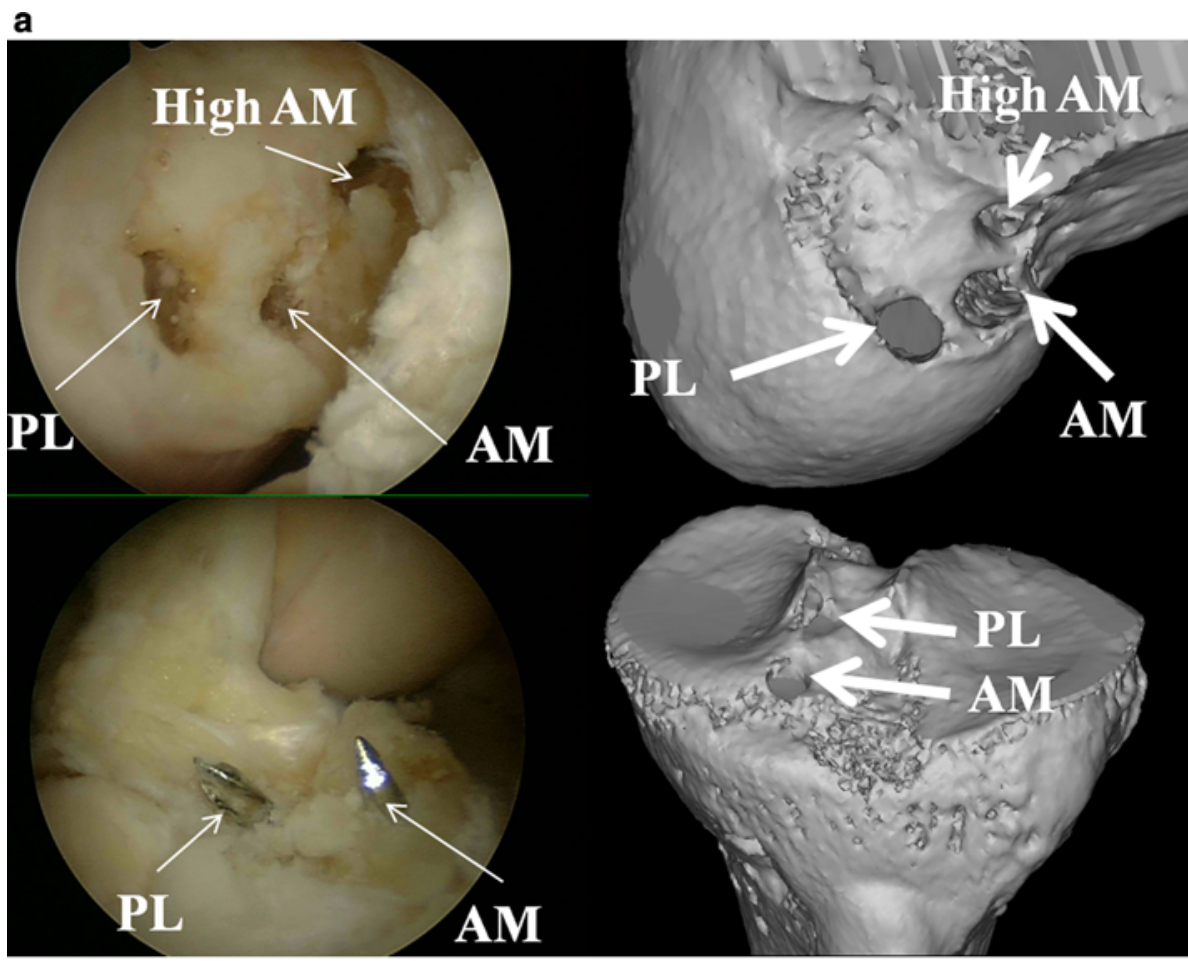

b

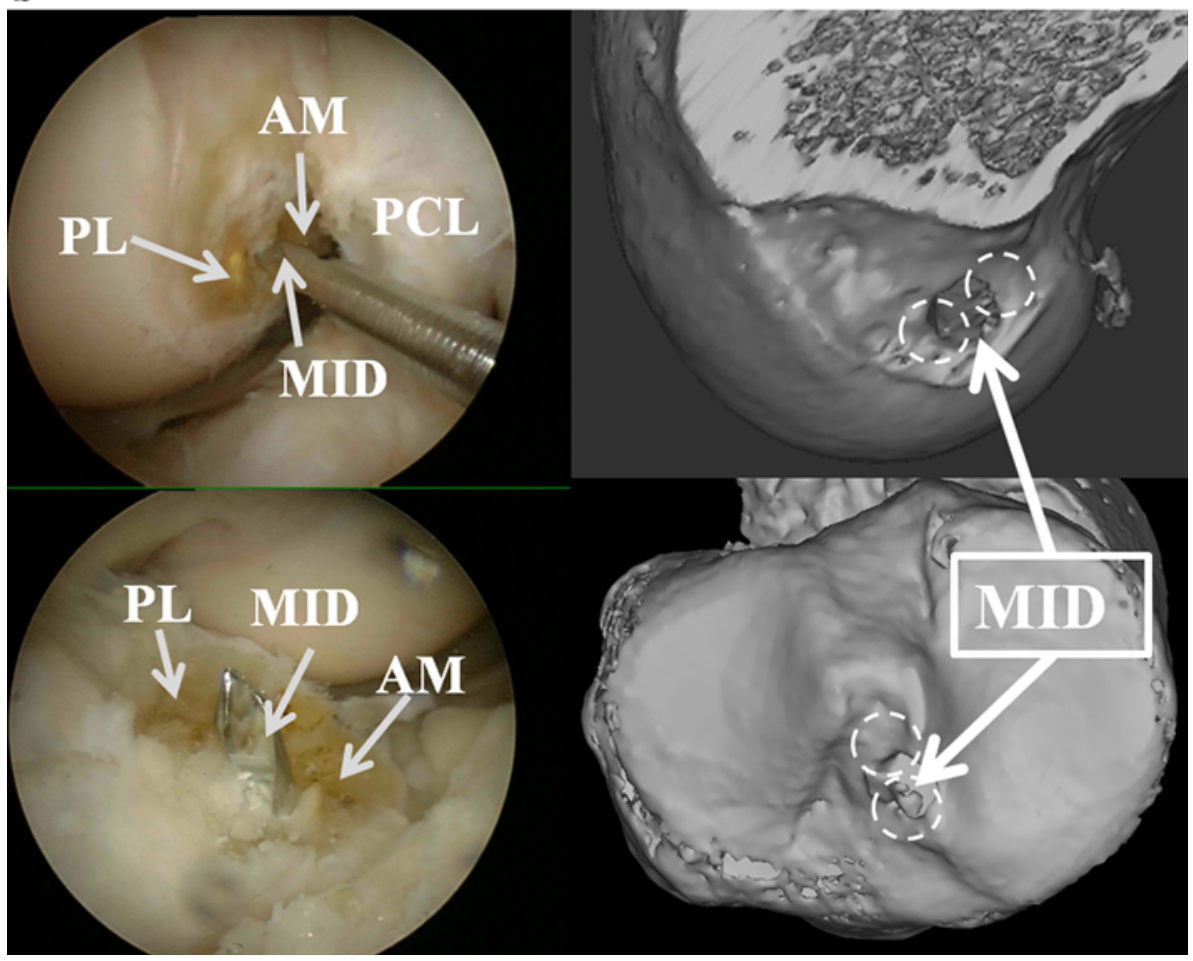

torque (simulated pivot-shift test) [20] at $0^{\circ}, 15^{\circ}, 30^{\circ}$ and $45^{\circ}$ of flexion. The anterior tibial load was as applied since the ACL is a major restraint to anterior tibial translation, and its application corresponds to the Lachman test and anterior drawer test. The force of $89 \mathrm{~N}$ is equivalent to that used in the KT-1000 arthrometer [10]. The combined valgus and internal tibial torque was chosen to simulate the pivot-shift test. While the external tibial loads were applied at each flexion angle, the 5-degree-of-freedom kinematics, forces, and moments of the intact knee were monitored. The ACL was transected arthroscopically, and the knee was tested with both modes of the testing system. Initially, 
the same loading conditions were employed in force control mode of the testing system to obtain kinematics of the ACL-deficient knee at each testing angle. Subsequently, in displacement-control mode, the identical path of motion of the intact knee was repeated in the ACL-deficient knee while new forces and moments of the specimen were recorded. By the principle of superposition, the vectorial difference in the measured forces between the intact and the ACL-deficient knee during the same path of motion gives the in situ force of the ACL [27, 33, 34].

All ACL reconstructions were performed sequentially. For the multiply reconstructed knees, the three ACL reconstructions, AM-AM, PL-PL and PL-high AM, were performed in random order. For the single reconstruction knees, only anatomic mid-bundle single-bundle reconstruction (MID-MID) was performed. The same graft (hamstring) and the same tibial fixation technique were used in all the reconstructions. External tibial loads were applied, and the kinematics of each reconstructed knee was analysed. After graft removal, the path of motion of the reconstructed knee at each testing angle was replayed while new forces and moments were monitored. The in situ force of the graft with each reconstruction technique was the vector difference in the measured forces between the reconstructed knee and the ACL-deficient knee with an identical path of motion.

\section{Statistical analysis}

Differences in ATT and in situ force at the different flexion angles were analysed using the Kruskal-Wallis test for comparison of all groups and the Mann-Whitney $U$ test between all the pairwise comparisons. A Bonferroni approach was used to adjust the alpha level for the pairwise post hoc comparisons, and statistical significance was assumed when $p<0.05$ for the Kruskal-Wallis test and $p<0.01$ for the Mann-Whitney $U$ test. Statistical analysis was performed using the software package SPSS version 17.0 (SPSS Inc., Chicago, IL, USA). Pairwise comparisons were made between the data of all reconstruction methods and the intact ACL and between the AM-AM, PL-PL and MID-MID reconstructions and the PL-high AM reconstruction.

\section{Results}

Anterior tibial translation during anterior load

The PL-high AM reconstructed knee had the largest anterior displacement with the anterior tibial load when compared with the other reconstructed knees at $0^{\circ}$ and
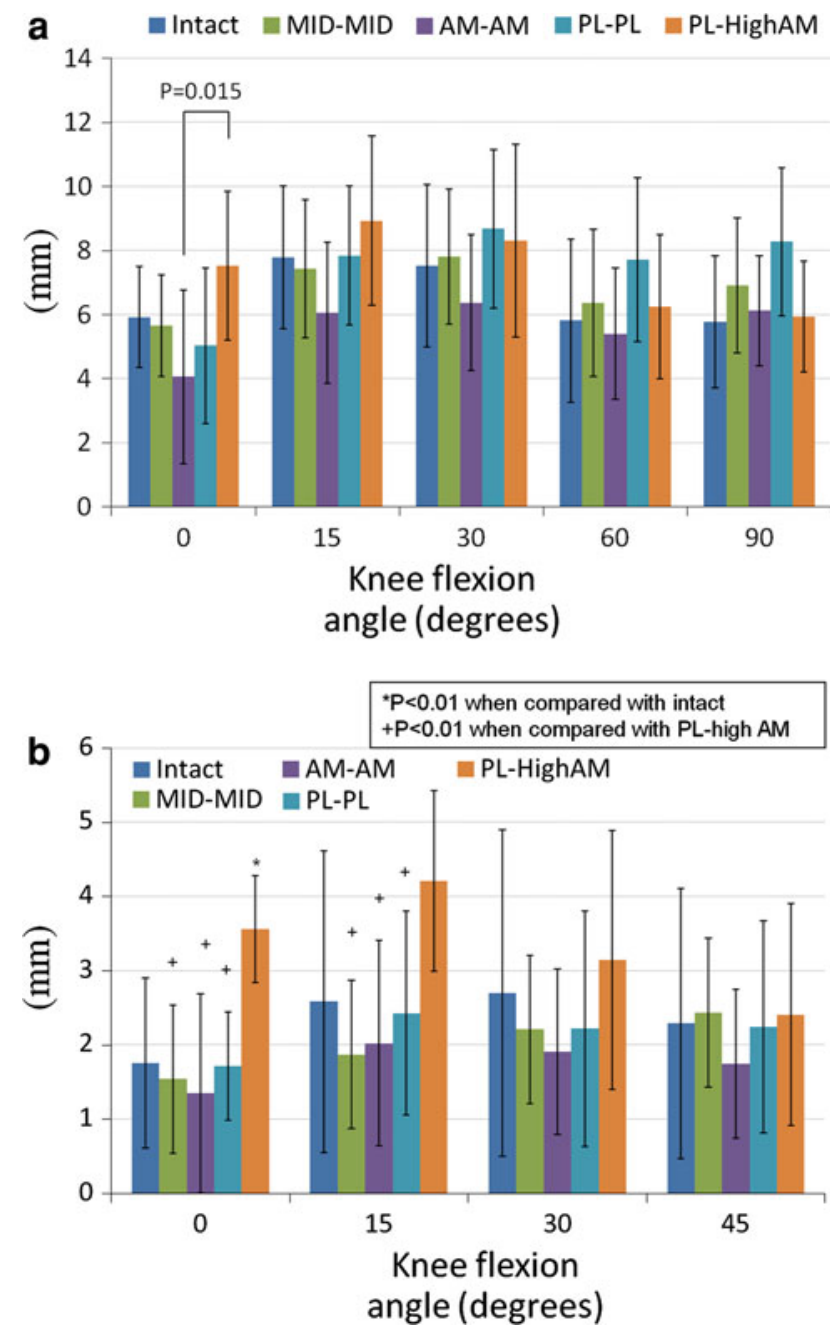

Fig. 4 a Anterior tibial translation (ATT) (mm) in response to the anterior tibial load. b Coupled anterior tibial translation (ATT) (mm) in response to the combined rotatory load

$15^{\circ}$ of knee flexion (Fig. 4a). At $30^{\circ}$ and $60^{\circ}$ of knee flexion, the PL-PL reconstructed knee had the largest displacement. However, overall there was no significant difference in ATT between the intact knees and the different reconstructions.

Coupled anterior tibial translation during the combined rotatory load

The PL-high AM reconstructed knee had a greater anterior displacement during the combined rotatory load than the intact knee at $0^{\circ}, 15^{\circ}$ and $30^{\circ}$ of knee flexion, although this difference was significant only at $0^{\circ}$ of knee flexion ( $p=0.001$ ) (Fig. 4b). The PL-high AM reconstructed knee had a significantly larger ATT at $0^{\circ}$ and $15^{\circ}$ of flexion under combined rotatory loading than the MID-MID $(p=$ $\left.0.003-0^{\circ}, 0.007-15^{\circ}\right), \mathrm{AM}-\mathrm{AM}\left(p=0.005-0^{\circ}, 0.005-15^{\circ}\right)$ and PL-PL $\left(p=0.001-0^{\circ}, 0.01-15^{\circ}\right)$ reconstructions. 

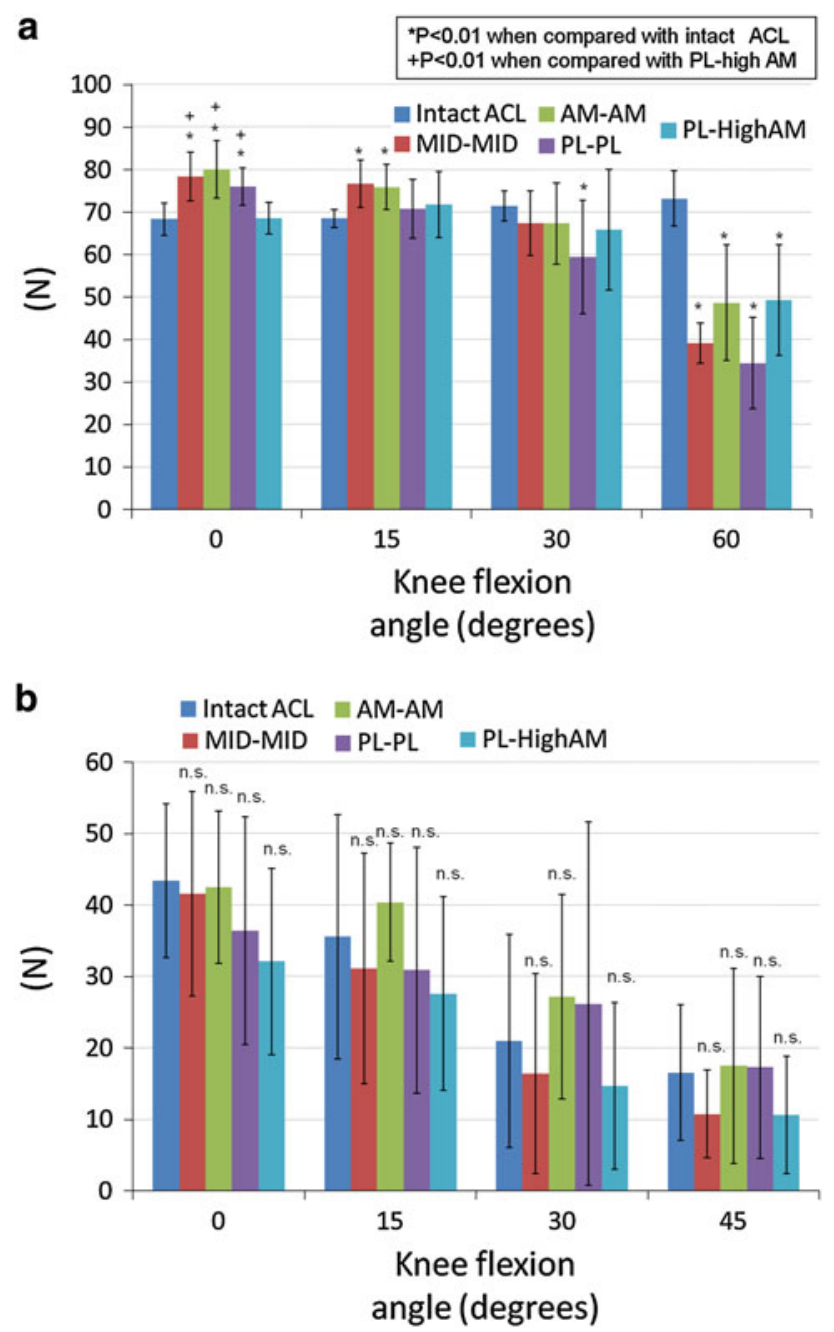

Fig. 5 a In situ forces (N) in response to the anterior tibial load. b In situ forces $(\mathrm{N})$ in response to the combined rotatory load

In situ forces during the anterior load

The MID-MID, AM-AM and PL-PL grafts had significantly higher in situ forces in response to the anterior load than the intact $\mathrm{ACL}$ at $0^{\circ}$ of knee flexion $(p=0.000,0.000$ and 0.001, respectively). The PL-high AM graft had a significantly lower in situ force at $0^{\circ}$ of knee flexion as compared to the MID-MID, AM-AM and PL-PL grafts ( $p=0.005,0.001$ and 0.005 , respectively) (Fig. 5a). The MID-MID and AM-AM grafts had significantly higher in situ forces than the intact $\mathrm{ACL}$ at $15^{\circ}$ of knee flexion ( $p=0.002$ and 0.002 , respectively). The PL-PL graft had a significantly lower in situ force than the intact ACL at $30^{\circ}$ and $60^{\circ}$ of knee flexion $(p=0.003$ and 0.000 , respectively). At $60^{\circ}$ of knee flexion, all grafts had a significantly lower in situ force when compared with the intact ACL $(p=0.000)$.
In situ forces during the combined rotatory load

The reconstructed grafts showed no significant difference from the intact ACL with respect to the in situ force under the combined rotatory loading (Fig. 5b). The PL-high AM had the lowest in situ force at $0^{\circ}, 15^{\circ}, 30^{\circ}$ and $45^{\circ}$ of knee flexion, whereas the AM-AM graft had the highest in situ force at $0^{\circ}, 15^{\circ}, 30^{\circ}$ and $45^{\circ}$ of knee flexion.

\section{Discussion}

In this study, different graft positions for single-bundle ACL reconstruction were compared to identify the position that best restores intact knee kinematics. The results show that anatomic reconstruction restores normal knee kinematics better than the non-anatomic ACL reconstruction.

The findings of graft isometry studies have supported femoral tunnel placement at a high position in the intercondylar notch in a non-anatomic location $[8,37]$, and often, the tibial tunnel must be placed slightly posteriorly to avoid roof impingement [17]. On the basis of these recommendations, the tibial tunnel is placed within the PL bundle of the ACL footprint [36]. Nevertheless, conventional singlebundle ACL reconstruction has consistently yielded high stability rates, high patient satisfaction and low revision rates, in the hands of experienced surgeons [21]. However, placement of the femoral tunnel lower on the clock face of the notch has been attracting growing attention $[18,22$, 26], which indicates that the anatomic placement of the femoral tunnel position is gaining favour. In a biomechanical cadaveric study, Loh et al. [22] found that grafts in the 10 o'clock position afforded better resistance to rotatory loads than grafts in the 11 o'clock position. The present study has shown that the conventional vertical reconstructed knee (PL-high AM) had a large ATT in response to an anterior load and the combined rotatory loads at $0^{\circ}, 15^{\circ}$ and $30^{\circ}$ of knee flexion, although the values were not statistically significant in all cases.

Steiner et al. [29] recommended that the tibial tunnel should be placed anterior and medial to the footprint, as long as it does not cause graft impingement [17]. The tibial tunnel positions of the AM-AM and MID-MID reconstructions are more anterior than that of the conventional (PL-high AM) and PL-PL reconstructions. Although these tunnels are placed more anteriorly and could potentially cause impingement [19], the presence of the lower femoral tunnel was expected to reduce this possibility [28].

Recent studies have shown that the optimal femoral and tibial insertion sites for an ACL graft are within the anatomic footprint of the ACL $[5,12]$. Some studies have shown that conventional single-bundle ACL reconstruction 
may be mechanically inferior to the native ACL position $[7,22,25]$, which suggests that the anatomic positioning of a single-bundle ACL reconstruction may provide better control of knee stability than non-anatomic positioning.

A recent clinical study demonstrated that placement of a posterior femoral tunnel and an anterior tibial tunnel afforded good results [25]. In the present study, the AMAM graft yielded a high in situ force in response to the external loads at all knee flexion angles. However, there is some concern about the increased re-injury rate because of the large strain in the AM-AM reconstructed graft. It should be noted that with all methods the graft had a lower in situ force than the intact ligament during ATT loading at higher flexion angles $\left(60^{\circ}\right)$.

The AM and PL bundles have a synergistic relationship [7], and the native ACL bundles do not function independently. The AM-AM reconstruction yielded better stability than any of the other reconstructions, and the MID-MID reconstruction did not have any major weaknesses. Steiner [29] recommended that the femoral tunnel be placed at the centre of the femoral footprint, although he recommended that the tibial tunnel be placed at the AM footprint.

Recent biomechanical studies have emphasized the importance of the PL bundle for constraining rotatory instability [36]. Contrary to previous findings, the results of the current study showed that the displacement of the AMAM reconstructed knee during the anterior tibial and the combined rotatory loading at $0^{\circ}, 15^{\circ}, 30^{\circ}$ and $45^{\circ}$ of knee flexion was less than that of the PL-PL reconstructed knee. However, the PL-PL reconstructed knee had less force than during the combined application of external loads at $0^{\circ}, 15^{\circ}$ and $30^{\circ}$ of knee flexion. These results suggest that the AM graft can play a role in rotatory stability in addition to the PL graft; however, the PL-PL reconstructed knee tended to have a higher ATT under anterior loading conditions at over $30^{\circ}$ of knee flexion, suggesting that the PL-PL tunnel position is not suitable for a single-bundle reconstruction.

This study has some limitations. The external loads applied in the present study were lower than those used in previous biomechanical studies [30,33,36]. In spite of this, it is believed that the controlled experimental study shows the same trends. The study used 6-mm-diameter grafts, whereas $9 \mathrm{~mm}$ or larger diameter grafts are used clinically in singlebundle ACL reconstructions. While the graft diameter does affect graft stiffness, it can be noted that the smaller grafts did restore the intact anterior tibial translation and in some cases the in situ force of the graft was greater than that of the intact ACL. Moreover, a recent study comparing 6- and 9-mm tunnels for a single-bundle ACL reconstruction revealed that increasing the graft size did not improve the time-zero biomechanical stability [4]. Grafts were made from both semitendinosus and gracilis tendons and there may be a difference in these tissues. In the present study, the femoral high AM tunnel, which is usually created using a trans-tibial technique, was created using a trans-portal technique, in order to avoid damage to the other the tibial tunnels. All the knee motions evaluated by the robotic system were static and at time zero, so the influence of healing could not be assessed. A sample-size analysis or post hoc power analysis was not performed and, despite the fact that statistically significant results were obtained, the large number of variables evaluated could increase the type 2 error. Although it cannot be claimed that the results of this study apply directly to singlebundle ACL reconstructions with larger grafts, it does emphasize the importance of anatomical tunnel position in knee biomechanics.

\section{Conclusion}

The in situ force and stability of the intact ACL was most closely reproduced by AM-AM single-bundle ACL reconstruction technique, as compared to the other ACL reconstruction techniques tested.

Acknowledgments The support of the Albert B. Ferguson, Jr. MD Orthopaedic Fund of The Pittsburgh Foundation is gratefully acknowledged. The authors would also like to thank Monica LindeRosen BS for all technical assistance and Paul Navrotski for CT scans.

Open Access This article is distributed under the terms of the Creative Commons Attribution License which permits any use, distribution, and reproduction in any medium, provided the original author(s) and the source are credited.

\section{References}

1. Arnoczky SP (1983) Anatomy of the anterior cruciate ligament. Clin Orthop Relat Res 28:19-25

2. Arnold MP, Kooloos J, van Kampen A (2001) Single-incision technique misses the anatomical femoral anterior cruciate ligament insertion: a cadaver study. Knee Surg Sports Traumatol Arthrosc 9:194-199

3. Bedi A, Altchek DW (2009) The "footprint" anterior cruciate ligament technique: an anatomic approach to anterior cruciate ligament reconstruction. Arthroscopy 25:1128-1138

4. Bedi A, Maak T, Musahl V, O'Loughlin P, Choi D, Citak M, Pearle AD (2011) Effect of tunnel position and graft size in single-bundle anterior cruciate ligament reconstruction: an evaluation of time-zero knee stability. Arthroscopy 27:1543-1551

5. Beynnon BD, Johnson RJ, Abate JA, Fleming BC, Nichols CE (2005) Treatment of anterior cruciate ligament injuries, part 2. Am J Sports Med 33:1751-1767

6. Biau DJ, Tournoux C, Katsahian S, Schranz P, Nizard R (2007) ACL reconstruction: a meta-analysis of functional scores. Clin Orthop Relat Res 458:180-187

7. Brophy RH, Voos JE, Shannon FJ, Granchi CC, Wickiewicz TL, Warren RF, Pearle AD (2008) Changes in the length of virtual anterior cruciate ligament fibers during stability testing: a 
comparison of conventional single-bundle reconstruction and native anterior cruciate ligament. Am J Sports Med 36:21962203

8. Cooper DE, Small J, Urrea L (1998) Factors affecting graft excursion patterns in endoscopic anterior cruciate ligament reconstruction. Knee Surg Sports Traumatol Arthrosc 6(Suppl 1):S20-S24

9. Diermann N, Schumacher T, Schanz S, Raschke MJ, Petersen W, Zantop T (2009) Rotational instability of the knee: internal tibial rotation under a simulated pivot shift test. Arch Orthop Trauma Surg 129:353-358

10. Fithian DC, Goltz DH, Funahashi TT (2003) Diagnosis of ligament injury part $\mathrm{C}$ instrumented laxity studies. In: Pedowitz RA, O'Corner JJ, Akeson WH (eds) Daniel's knee injuries: ligament and cartilage structure, function, injury, and repair. Lippincott Williams and Wilkins, Philadelphia, pp 370-408

11. Fleming BC, Abate JA, Peura GD, Beynnon BD (2001) The relationship between graft tensioning and the anterior-posterior laxity in the anterior cruciate ligament reconstructed goat knee. J Orthop Res 19:841-844

12. Fu FH, Bennett CH, Ma CB, Menetrey J, Lattermann C (2000) Current trends in anterior cruciate ligament reconstruction. Part II. Operative procedures and clinical correlations. Am J Sports Med 28:124-130

13. Fu FH, Shen W, Starman JS, Okeke N, Irrgang JJ (2008) Primary anatomic double-bundle anterior cruciate ligament reconstruction: a preliminary 2-year prospective study. Am J Sports Med 36:1263-1274

14. Girgis FG, Marshall JL, Monajem A (1975) The cruciate ligaments of the knee joint. Anatomical, functional and experimental analysis. Clin Orthop Relat Res 106:216-231

15. Harter RA, Osternig LR, Singer KM, James SL, Larson RL, Jones DC (1988) Long-term evaluation of knee stability and function following surgical reconstruction for anterior cruciate ligament insufficiency. Am J Sports Med 16:434-443

16. Hoher J, Kanamori A, Zeminski J, Fu FH, Woo SL (2001) The position of the tibia during graft fixation affects knee kinematics and graft forces for anterior cruciate ligament reconstruction. Am J Sports Med 29:771-776

17. Howell SM (1998) Principles for placing the tibial tunnel and avoiding roof impingement during reconstruction of a torn anterior cruciate ligament. Knee Surg Sports Traumatol Arthrosc 6(Suppl 1):S49-S55

18. Howell SM, Gittins ME, Gottlieb JE, Traina SM, Zoellner TM (2001) The relationship between the angle of the tibial tunnel in the coronal plane and loss of flexion and anterior laxity after anterior cruciate ligament reconstruction. Am J Sports Med 29:567-574

19. Iriuchishima T, Tajima G, Ingham SJ, Shen W, Horaguchi T, Saito A, Smolinski P, Fu FH (2009) Intercondylar roof impingement pressure after anterior cruciate ligament reconstruction in a porcine model. Knee Surg Sports Traumatol Arthrosc 17:590-594

20. Kanamori A, Woo SL, Ma CB, Zeminski J, Rudy TW, Li G, Livesay GA (2000) The forces in the anterior cruciate ligament and knee kinematics during a simulated pivot shift test: a human cadaveric study using robotic technology. Arthroscopy 16:633-639

21. Lewis PB, Parameswaran AD, Rue JP, Bach BR Jr (2008) Systematic review of single-bundle anterior cruciate ligament reconstruction outcomes: a baseline assessment for consideration of double-bundle techniques. Am J Sports Med 36:2028-2036
22. Loh JC, Fukuda Y, Tsuda E, Steadman RJ, Fu FH, Woo SL (2003) Knee stability and graft function following anterior cruciate ligament reconstruction: comparison between 11 o'clock and 10 o'clock femoral tunnel placement. 2002 Richard O'Connor Award paper. Arthroscopy 19:297-304

23. Lyman S, Koulouvaris P, Sherman S, Do H, Mandl LA, Marx RG (2009) Epidemiology of anterior cruciate ligament reconstruction: trends, readmissions, and subsequent knee surgery. J Bone Joint Surg Am 91:2321-2328

24. Martins CAQ, Kropf EJ, Shen W, van Eck CF, Fu FH (2008) The concept of anatomic anterior cruciate ligament reconstruction. Oper Tech Sports Med 16:104-115

25. Moisala AS, Jarvela T, Harilainen A, Sandelin J, Kannus P, Jarvinen M (2007) The effect of graft placement on the clinical outcome of the anterior cruciate ligament reconstruction: a prospective study. Knee Surg Sports Traumatol Arthrosc 15:879-887

26. Musahl V, Plakseychuk A, VanScyoc A, Sasaki T, Debski RE, McMahon PJ, Fu FH (2005) Varying femoral tunnels between the anatomical footprint and isometric positions: effect on kinematics of the anterior cruciate ligament-reconstructed knee. Am J Sports Med 33:712-718

27. Rudy TW, Livesay GA, Woo SL, Fu FH (1996) A combined robotic/universal force sensor approach to determine in situ forces of knee ligaments. J Biomech 29:1357-1360

28. Shen W, Forsythe B, Ingham SM, Honkamp NJ, Fu FH (2008) Application of the anatomic double-bundle reconstruction concept to revision and augmentation anterior cruciate ligament surgeries. J Bone Joint Surg Am 90(Suppl 4):20-34

29. Steiner ME, Battaglia TC, Heming JF, Rand JD, Festa A, Baria M (2009) Independent drilling outperforms conventional transtibial drilling in anterior cruciate ligament reconstruction. Am J Sports Med 37:1912-1921

30. Vercillo F, Woo SL, Noorani SY, Dede O (2007) Determination of a safe range of knee flexion angles for fixation of the grafts in double-bundle anterior cruciate ligament reconstruction: a human cadaveric study. Am J Sports Med 35:1513-1520

31. Wolf EM (ed) (2001) Arthroscopic anterior cruciate ligament reconstruction: Trans-Fix technique using doubled semitendinosus and gracilis tendons. Springer, Berlin

32. Woo SL, Wu C, Dede O, Vercillo F, Noorani S (2006) Biomechanics and anterior cruciate ligament reconstruction. J Orthop Surg Res 1:2

33. Wu C, Noorani S, Vercillo F, Woo SL (2009) Tension patterns of the anteromedial and posterolateral grafts in a double-bundle anterior cruciate ligament reconstruction. J Orthop Res 27:879-884

34. Yagi M, Wong EK, Kanamori A, Debski RE, Fu FH, Woo SL (2002) Biomechanical analysis of an anatomic anterior cruciate ligament reconstruction. Am J Sports Med 30:660-666

35. Yasuda K, Ichiyama H, Kondo E, Miyatake S, Inoue M, Tanabe Y (2008) An in vivo biomechanical study on the tension-versusknee flexion angle curves of 2 grafts in anatomic double-bundle anterior cruciate ligament reconstruction: effects of initial tension and internal tibial rotation. Arthroscopy 24:276-284

36. Zantop T, Herbort M, Raschke MJ, Fu FH, Petersen W (2007) The role of the anteromedial and posterolateral bundles of the anterior cruciate ligament in anterior tibial translation and internal rotation. Am J Sports Med 35:223-227

37. Zavras TD, Race A, Bull AM, Amis AA (2001) A comparative study of 'isometric' points for anterior cruciate ligament graft attachment. Knee Surg Sports Traumatol Arthrosc 9:28-33 